\title{
Case report on Ewing's sarcoma with review of literature
}

\author{
Sonam Agrawal', Vinita C Rathod ${ }^{2}$, Pankaj Kumar Agrawal $^{3}$, Swati Saluja ${ }^{4}$ \\ ${ }^{1,4}$ Post graduate student, ${ }^{2}$ Professor and Head, Department of Oral and Maxillofacial Pathology, Rungta College of Dental Sciences and \\ Research. Bhilai, Chhattisgarh, ${ }^{3}$ Senior Lecturer, Department of Oral and Maxillofacial Pathology, Maitri College of Dentistry and Research \\ Center, Anjora, Durg, Chhattisgarh
}

\section{A B S T R A C T}

Ewings sarcoma is a highly malignant primary mesenchymal neoplasm of bone, which was first reported in 1921 by Ewing. The exact cell of origin of Ewing Sarcoma is not known it is generally believed that the lesion arises from either the endothelial cells of the blood vessels within the bone or from the undifferentiated reticulo-endothelial cell. Primitive neuroectodermal tumors (PNETs) arises from mesenchymal stem cells that differentiates along neural cell lineage. Reports of Ewings sarcoma constitute about $10 \%$ of all malignant bone tumor. A case of Ewings sarcoma in 10 year old male patient is reported, who visited to Dept of Oral and Maxillo-facial Pathology in Rungta College of Dental Sciences And Research, with chief complaint of pain and swelling in right side of face since 1 month. Early diagnosis of this case helps in selecting the appropriate treatment procedure and to avoid further complication.

Key words: Ewings Sarcoma, PNET, Immuno-histochemistry
Access this article online

Website:

http://nepjol.info/index.php/AJMS

DOI: 10.3126/ajms.v6i6.12283

\section{INTRODUCTION}

Ewings sarcoma is a highly malignant primary mesenchymal neoplasm of bone, which was first reported in 1921 by Ewing. The exact cell of origin of Ewing Sarcoma is not known it is generally believed that the lesion arises from either the endothelial cells of the blood vessels within the bone or from the undifferentiated reticulo-endothelial cell. Primitive neuroectoderrmal tumors arise from mesenchymal stem cells that differentiate along neural cell lineage. Reports of Ewings sarcoma constitute about 10\% of all malignant bone tumor.

Primitive neuroectodermal tumors (PNETs) are a group of highly malignant tumors composed of small round cells of neuroectodermal origin that affect soft tissue and bone. They exhibit great diversity in their clinical manifestations and pathologic similarities with other small, round cell tumors. This has made classifying this family of tumors challenging and controversial. Batsakis et al (1996) divided the primitive neuroectodermal tumor (PNET) family of tumors into the following 3 groups based on the tissue of origin:
- CNS primitive neuroectodermal tumors (PNETs) - Tumors derived from the central nervous system

- Neuroblastoma - Tumors derived from the autonomic nervous system

- Peripheral primitive neuroectodermal tumors (pPNETs) - Tumors derived from tissues outside the central and autonomic nervous system.

- We report the instance of Ewings Sarcoma in 10 year old male patient, who visited to Department of Oral and Maxillo-facial Pathology in Rungta College of Dental Sciences And Research, with chief complaint of pain and swelling in right side of face since 1 month.

\section{CASE REPORT}

A 10 year old male patient visited to Dept of Oral and Maxillo-facial Pathology in Rungta College of Dental Sciences And Research,with chief complaint of pain and swelling in right side of face since one month. 
The history revealed that patient was apparently alright before six months then he had trauma during interpersonal violence over the zygomatic region. Since then he was having swelling over there.

The swelling regressed after taking primary aids. The swelling became enlarged and associated with pain since two months.

Extra orally the swelling was single large oval swelling, normal skin in color measuring about $3 \times 3 \times 3 \mathrm{~cm}$ in diameter was present extending superiorly from corner of the eye to corner of mouth inferiorly, medially to lateral side of nose, obliterating naso-labial fold to zygomatic arch laterally (Figure 1).

Intraoral the swelling was extending from buccal aspect of right Maxillary teeth upto right maxillary tuberosity and bluish red in colour. Blood was oozes from the swelling (Figure 2).

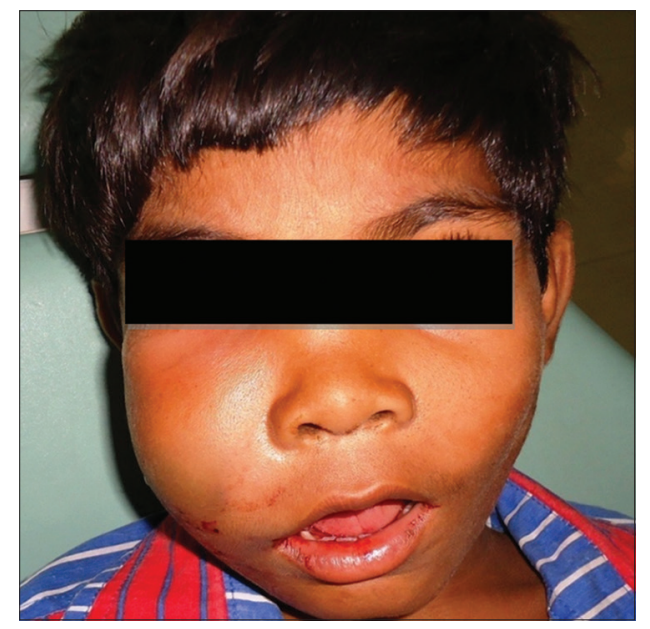

Figure 1 : Extra oral swelling

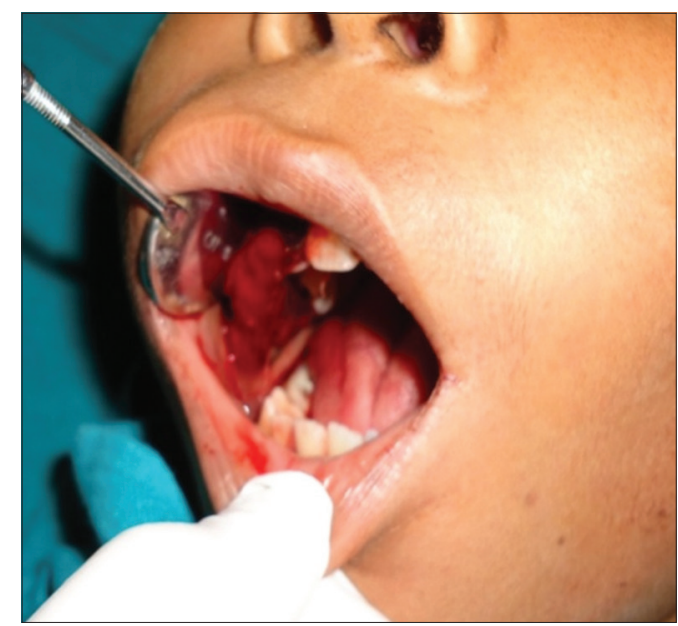

Figure 2 : Intraoral swelling
Right submandibular lymph node was tender, firm in consistency and mobile. Provisional diagnosis was made Sarcoma with secondary infection. After radiographic examination CT scan shows small polypoidal lesion with internal areas of new bone formation noted involving of maxilla with involvement of right maxillary sinus. (Figure 3)

Erosion of medial wall of sinus is seen bulging into right side nasal cavity, posteriorly into infratemporal fossa, anteriorly into the soft tissue of cheek. Inferiorly there is involvement of alveolar margins associated with large overlying swelling on right side of face.

The mass has also infilterated into the right orbit with involvement of retrobulbar fat and displacing the eyeball.

The macroscopic appearance of incisional biopsy specimen was brownish grey in colour and measuring about 1 x.5 cm in size. (Figure 4.1) The histopathologic report revealed

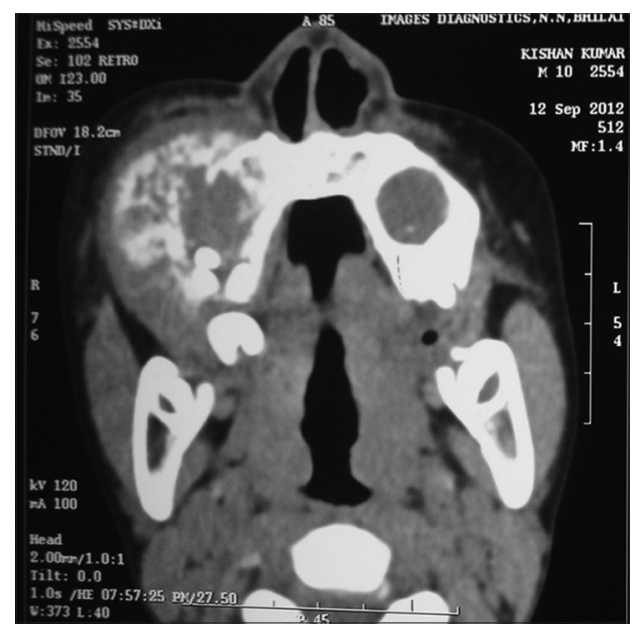

Figure 3: CT Scan showing dense polyploidal lesion with internal areas of new bone formation involving maxilla with involvement of right maxillary sinus

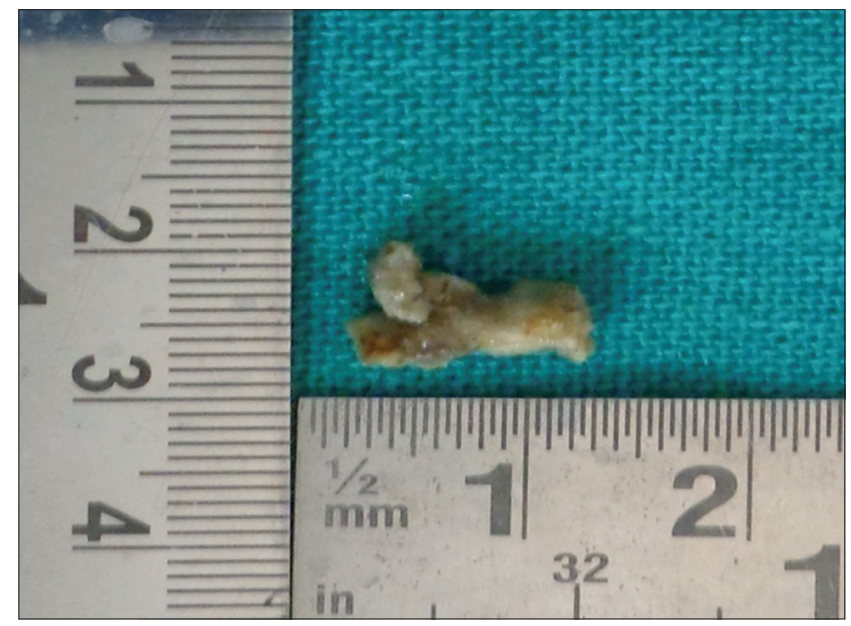

Figure 4.1: Greyish white irregular firm mass of size $1.5 \times .5 \times .5 \mathrm{~cm}$ 
hap-hazard proliferation of ulcerated overlying stratified squamous epithelium. Underlying connective tissue stroma is hypercellular with numerous round small capillaries and around capillaries lobular architectural arrangement of numerous round to oval cells with dark nucleus and eosinophilic cytoplasm.

Few cells are acute inflammatory type. Numerous vascular space with extravasation and with melanin pigment (Figure 4.2, 4.3, 4.4, 4.5, 4.6).

Around these total structure numerous proliferated bundles of neural tissue appreciated (Figure 4.7).

Based on the above mentioned features the histopathological diagnosis was made as Round cell tumor.

Differential diagnosis: Ewings Sarcoma, Rhabdo myosarcoma,PNET, Burkit Lymphoma

To confirm the diagnosis.IHC was performed. Immunohistochemistry showed positivity for CD99, CD45

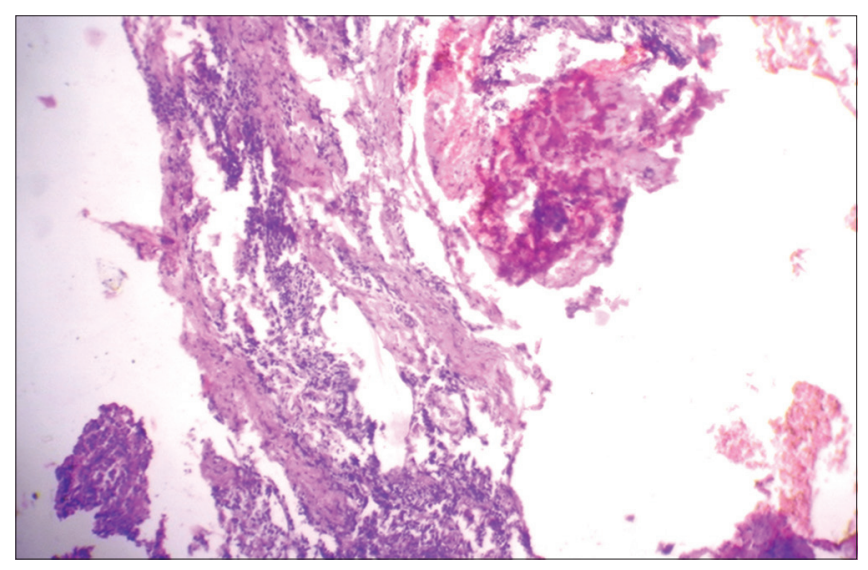

Figure 4.2: ( H\&E, 4x) Photomicrophotograph showing hypercellular CT stroma.

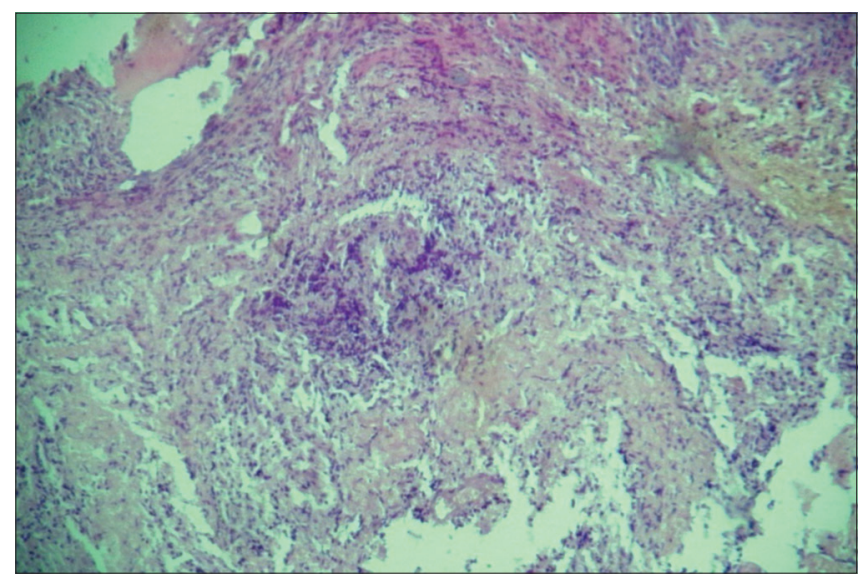

Figure 4.3: (H \& E, 10x ) Microphotograph showing hypercellular CT stroma. and was negative for desmin, then confirmed diagnosis of Ewings Sarcoma was made (Figure 5.1, 5.2, 5.3).

\section{DISCUSSION}

James Ewing (1866-1943) first described the tumor, establishing that the disease was different from lymphoma

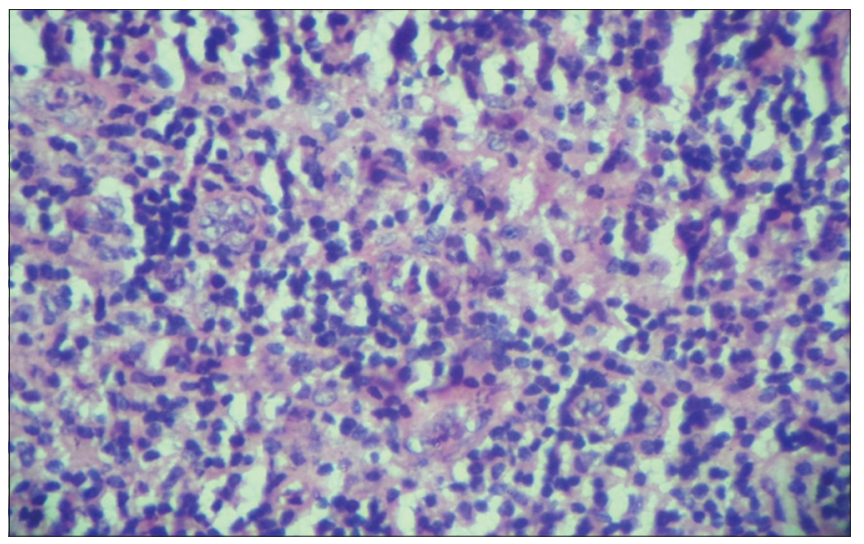

Figure 4.4: (H \& E, 40x) Microphotograph showing hypercellular CT stroma with round to oval blue cells, hyperchromatic giant nucleus, with few signs of anaplasia and scanty eosinophilic cytoplasm.

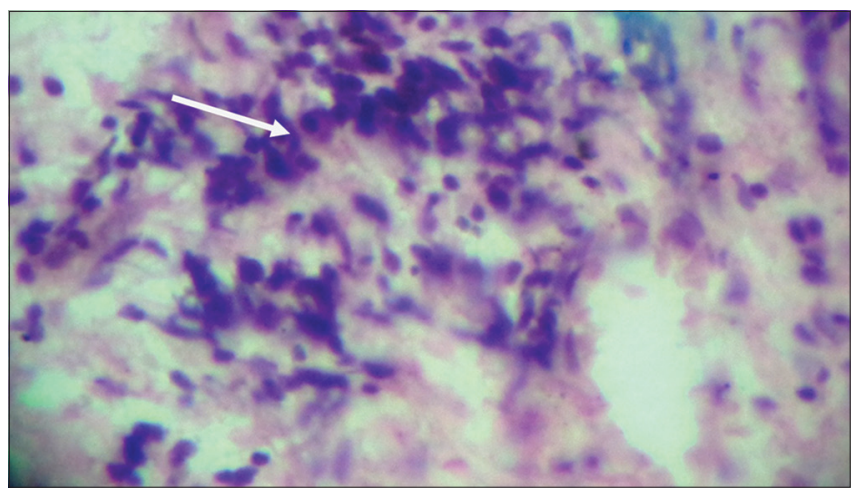

Figure 4.5: (H \& E, 40x) Microphotograph showing filigri pattern

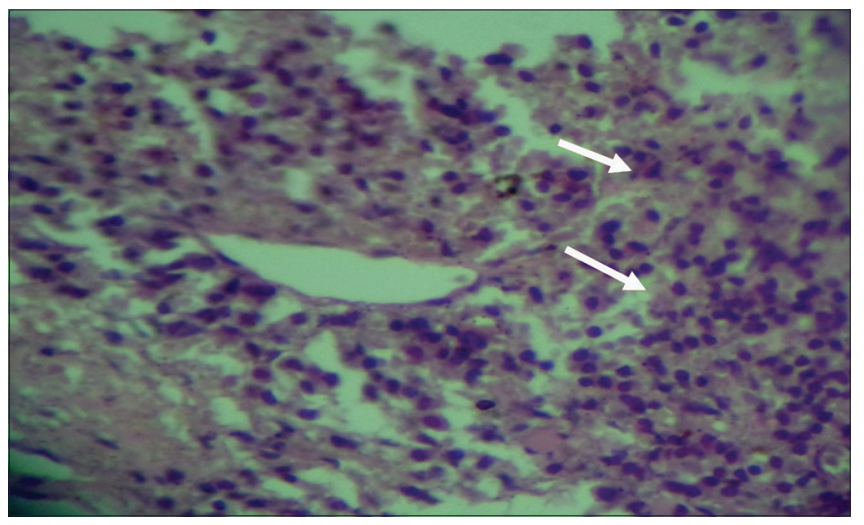

Figure 4.6: Photomicrophotograph showing hypercellular CT stroma with round to oval blue cells, hyperchromatic giant nucleus, melanin pigmentation with few signs of anaplasia and scanty eosinophilic cytoplasm. 


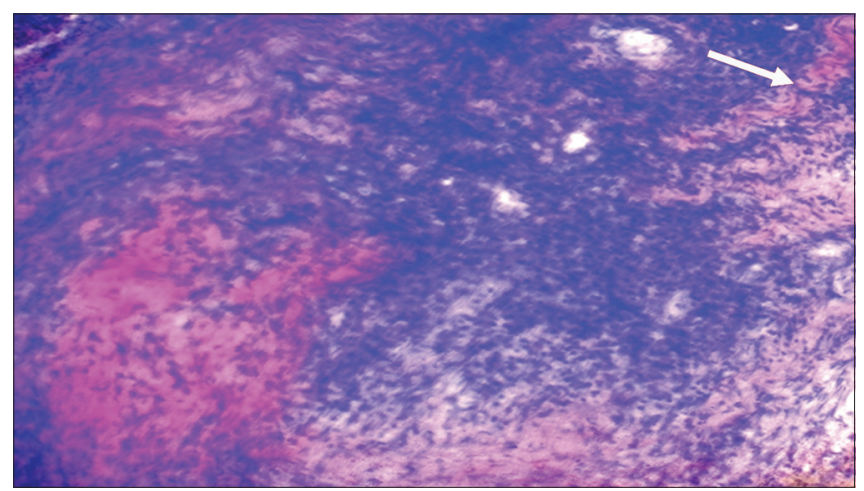

Figure 4.7: (H\&E ,10x) Photomicrophotograph showing hypercellular CT stroma with neural bundles, numerous blue cells.

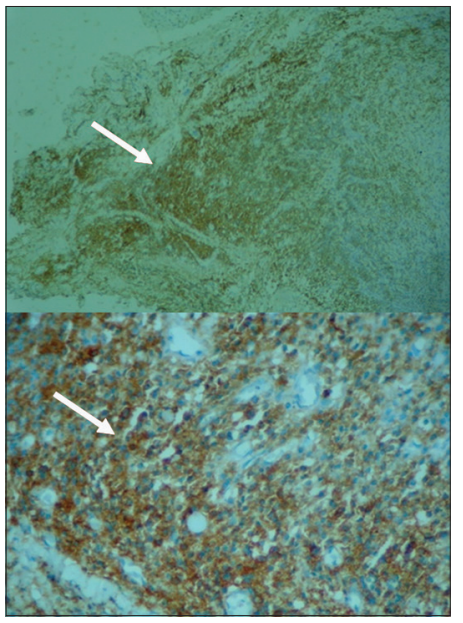

Figure 5.1: Immunohistochemistry, strongly positive for CD99

and other types of cancer known at that time. In 1921, he described a lethal primary bone lesion that affects children and young adults and most frequently originates in the long bones $(47 \%)$, pelvis $(19 \%)$ or ribs $(12 \%)$. The skull is rarely involved, probably in less than $4 \%$ of the cases, with the frontal and parietal bones being the most commonly affected.

Extraosseous Ewing's sarcoma (EES) has been recognized as a distinct disease entity that afflicts young adults in the second and third decades of life, with equal sex predilection. The EES commonly involves the paravertebral regions of the spine and in rare instances, these lesions arise in the intracranial compartment, where they have been commonly misdiagnosed as c-PNET, because of the similarity in their histological appearance.

Very few cases of the central nervous system extraosseous Ewing's sarcoma (CNS-EES) have been reported in pathology literature.Jay et al. was probably the first to describe a patient with an isolated posterior fossa mass that histologically resembled a medulloblastoma, but demonstrated the $\mathrm{t}(11 ; 22)$ (q24;q12) translocation, which confirmed CNS-EES. As far as our knowledge goes, this is the seventh case we are reporting.

Asian Journal of Medical Sciences | Nov-Dec 2015 | Vol 6 | Issue 6

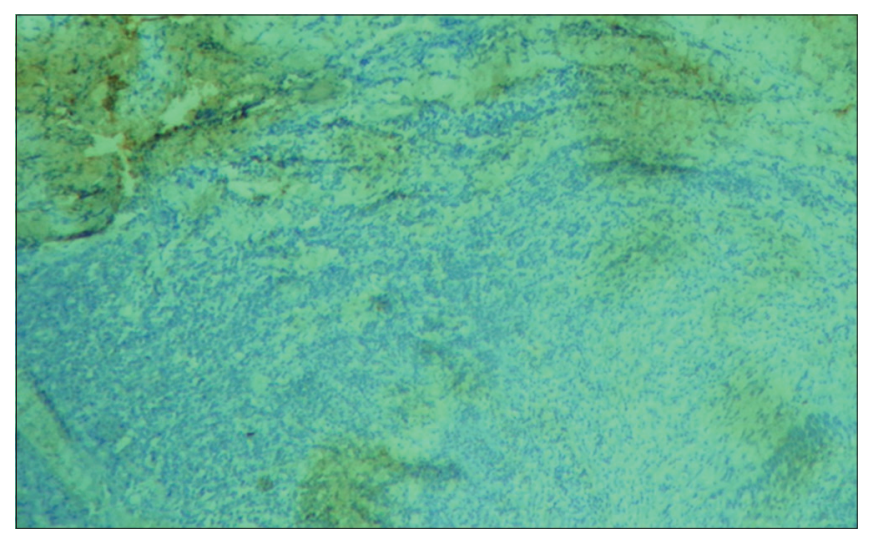

Figure 5.3: Immunohistochemistry negative for desmin

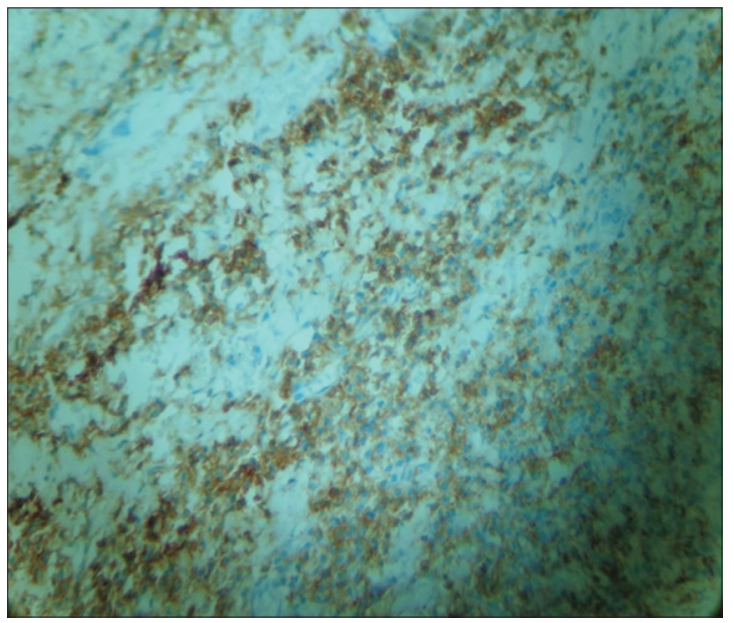

Figure 5.2: Immunohistochemistry, positive for CD 45

Diagnosis requires a histopathological examination, immunohistochemistry, and cytogenetics. The differential diagnosis of an intracranial round cell tumor is primitive neuroectodermal tumor (neuroblastoma), lymphoma, rhabdomyosarcoma, and Ewing's sarcoma.

The histological examination reveals that these tumors are composed of small, undifferentiated neuroectodermal cells and frequently demonstrate immunohistochemical and/or electron microscopic features of glial or neuronal differentiation. Recent advances in the molecular classification has allowed a clear pathological distinction between c-PNET and CNS-EES. CNS-EES is known to demonstrate in $97 \%$ of the patients, a strong membrane expression of the MIC-2 gene product, designated CD99, which is specifically recognized by the monoclonal antibodies $\mathrm{O} 13$ and HBA71. In addition, the chromosomal translocation $\mathrm{t}(11,22)(\mathrm{q} 24 ; \mathrm{q} 12)$, detected by FISH, is found in more than $90 \%$ of EES. This nonrandom translocation is not found in the central primitive neuroectodermal tumors (c-PNET) such as the medulloblastoma and supratentorial PNET. 
Ewing's sarcoma (ES) is a rare malignant small round cell tumor that primarily affects the skeletal system. It accounts for 4 to $10 \%$ of all types of bone cancer, with long bones and pelvis being the most common locations. It affects mainly adolescents and young adults and is rarely seen before the age of 5 and after the age of 30 .

Clinically, this tumor has an aggressive behavior characterized by rapid growth and high probability of micrometastasis at diagnosis.

ES is a malignant neoplasm that primarily affects long bones of the extremities with nearly $50 \%$ of reported cases involving the femur and pelvis. It exhibits a marked predilection for whites and is rarely seen among blacks.

The majority of the patients affected are between the ages 5 and 20, whereas the disease is distinctly uncommon in individuals before age 5 and after age 30 (Braz Dent). ES arising from the bones of the head and neck region is exceedingly uncommon. When it occurs in the jaw, mandible is more frequently affected than the maxilla.(8:1)

But in our case it has occurred in Maxilla.

Molecular diagnosis of sarcomas

\begin{tabular}{lc}
\hline Tumor & Translocation \\
\hline Ewing/PNET & $\mathrm{t}(11 ; 22)(\mathrm{q} 24 ; \mathrm{q} 12)$ \\
& $\mathrm{t}(21 ; 22)(\mathrm{q} 22 ; \mathrm{q} 12)$ \\
Alveolar rhabdomyosarcoma & $\mathrm{t}(2 ; 13)(\mathrm{q} 35 ; \mathrm{q} 14)$ \\
& $\mathrm{t}(1 ; 13)(\mathrm{p} 36 ; \mathrm{q} 14)$ \\
Desmoplastic small round cell tumor & $\mathrm{t}(11 ; 22)(\mathrm{p} 13 ; \mathrm{q} 12)$ \\
Synovial sarcoma & $\mathrm{t}(\mathrm{X} ; 18)(\mathrm{p} 11.2 ; \mathrm{q} 11.2)$ \\
Congenital fibrosarcoma & $\mathrm{t}(12 ; 15)(\mathrm{p} 13 ; \mathrm{q} 25)$ \\
Clear cell sarcoma & $\mathrm{t}(12 ; 22)(\mathrm{q} 13 ; \mathrm{q} 12)$ \\
\hline
\end{tabular}

(Angervall L, Enzinger FM. Extraskeletal neoplasm resembling Ewing's sarcoma. Cancer. 1975;36:240-51)

\begin{tabular}{lcccc}
\hline IHC & $\begin{array}{c}\text { Ewings } \\
\text { sarcoma }\end{array}$ & PNET & Rhabdomyosarcoma & Lymphoma \\
\hline CD99 & ++ & + & - & - \\
NSE & - & + & Rarely present & - \\
PGP 9.5 & - & + & - & - \\
S 100 & - & + & Rarely present & - \\
Leu 7 & - & + & - & - \\
CD 45 & + & + & - & ++ \\
Desmin & - & - & + & - \\
\hline
\end{tabular}

The present case was positive for CD 99, CD45, and negative for desmin immunomarkers, leading to a diagnosis of ES. Ewing's sarcoma is usually sensitive to chemotherapy and radiotherapy. Modern treatments are based on combined modality of treatment: local therapy (surgery and/or radiotherapy to the main tumor) followed by chemotherapy(for management of micrometastasis).

\section{CONCLUSION}

The diagnosis of EFT amalgamates the usual or classical tools such as histology and immunohistochemistry with newer molecular technologies like FISH and PCR. The goal of these is to furnish a correct diagnosis and give sufficient information about the tumor that would aid in better risk assessment, improve clinical management, and survival of the patients.

\section{REFERENCES}

1. Stout AP. A tumor of the ulnar nerve. Proc NY Pathol Soc. 1918; 12:2-12.

2. Ewing J. Diffuse endothelioma of bone. Proc NY Pathol Soc 1921;21:17-24.

3. Angervall $\mathrm{L}$ and Enzinger FM. Extraskeletal neoplasm resembling Ewing's sarcoma. Cancer 1975; 36:240-251.

4. Jaffe R, Santamaria M, Yunis EJ, Tannery NH, Agostini RM, Medina J, et al. The neuroectodermal tumor of bone. Am J Surg Pathol 1984; 8:885-898.

5. Gurney JG, Swensen AR and Bulterys M. Malignant bone tumors. In: Ries LA, et al., editors. Cancer incidence and survival among children and adolescents: United States SEER Program 1975-1995.

6. Neville D, Damn DD, Allen CM and Bouquot JE. Oral And Maxillofacial Pathology. 3rd ed. Philadelphia: WB Saunders; 2009. Hematologic Disorders; pp. 604-607.

7. Sharda P, Girish HC and Priya NS. Ewings sarcoma of mandible. J. Oral Maxillo facial pathology 2006; 10(1):31-35.

8. Mark RE and Stern D. Oral and Maxillofacial Pathology. A rational for diagnosis and treatment. Malignant neoplasm of bone;807.

9. Devita, Hellman and Rosenberg. Cancer 'of childhood. Cancer principles and practice of oncology ( $8^{\text {th }}$ edition) 2008,2:2061

10. Shafers, Hine and Levy. Benign and malignant tumors of the oral cavity. Shafers Text book of oral pathology ( $6^{\text {th }}$ edition) 2009,165 